\title{
CLINICAL IMPORTANCE OF ENDOSCOPIC MIDDLE EAR DISSECTION ON CADAVER
}

\author{
Umesh Prasad Sinha1, Dhirendra Kumar Singh ${ }^{2}$
}

${ }^{1}$ Assistant Professor, Department of Anatomy, Government Medical College, Bettiah.

2 Professor and HOD, Department of Anatomy, Government Medical College, Bettiah.

\section{ABSTRACT}

\section{BACKGROUND}

In recent years use of endoscope for diagnostic and surgical procedures is increasing in ENT practice due to clear and wide vision of anatomical structures. However, there are some difficulties with use of endoscope because one hand remains engaged in holding endoscope and dissection should be carried out with one hand only on viewing the monitor.

The aims and objectives of this study is to assess the difficulties during dissection and visualise the different structures of middle ear cavity.

\section{MATERIALS AND METHODS}

During 2014 to 2016, 34 temporal bone were dissected with endoscopic vision. We used 0 degrees to 45 degrees and $4 \mathrm{~mm}$ endoscope.

\section{RESULTS}

After initial difficulties of holding of endoscope with left hand and doing dissection with right hand on viewing the monitor, there was no major technical difficulty later on. We were able to see middle ear structures with minimal dissection.

\section{CONCLUSION}

The hand control of holding endoscope and doing dissection on viewing monitor was adopted very soon and there was wide and clear view of middle ear structures.

\section{KEYWORDS}

Clinical Importance, Endoscopic, Middle Ear Dissection, Cadaver.

HOW TO CITE THIS ARTICLE: Sinha UP, Singh DK. Clinical importance of endoscopic middle ear dissection on cadaver. J. Evolution Med. Dent. Sci. 2017;6(19):1534-1537, DOI: 10.14260/Jemds/2017/337

\section{BACKGROUND}

Today the surgical procedures of the middle ear are traditionally carried out with the help of operating microscope. In spite of some benefits, because of its restricted and linear view field, the microscope does not allow a proper viewing of some spaces of middle ear. Exposure for viewing these areas often requires wide drilling of mastoid either by retroauricular or endaural incision. It disturbs the normal anatomy of middle ear, which gives discomfort after surgery and even after such wide exploration some retrotympanic spaces is out of vision by operating microscope. Such type of challenges requires alternative instruments for ear surgery. Endoscopic ear surgery is nowadays has become popular, because it has ability to fulfil such type of requirements. Although, the endoscopes are already used in our speciality for at least 15 years, in otology use of such instruments is still very uncommon. One of the main reasons may be the role of prominent otologists who indicate the use of endoscope only for secondary procedures and diagnosis. $1,2,3,4$

Financial or Other, Competing Interest: None.

Submission 27-01-2017, Peer Review 21-02-2017,

Acceptance 27-02-2017, Published 06-03-2017.

Corresponding Author:

Dr. Umesh Prasad Sinha,

\#77, LIC Colony,

Po-Lohianagar

Kankar Bagh,

Patna-20.

E-mail: umesh18163@yahoo.co.in

DOI: $10.14260 /$ jemds $/ 2017 / 337$

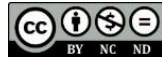

\section{Aims and Objectives}

The aims and objectives of this study was to become familiar with use of endoscope for the exploration of middle ear cavity. With the use of endoscope, the structure of middle ear was viewed on monitor. So, some practice was required for coordination of hands for dissection. After regular dissection, left hand was adjusted for holding endoscope as well as dissecting the tissue with right hand.

The endoscopic anatomy of middle ear was so clear and vision was so wide and extensive that anatomical structures was clearly visualised with minimal dissection. So, it helps to understand proper anatomy of middle ear cavity and gives extra confidence to otologists during ear surgery.

\section{MATERIALS AND METHODS}

1. Wet temporal bone.

2. Endoscope ( 0 degree and 45 degrees, $4 \mathrm{~mm}$ wide and 18 cm long).

3. Endoscopic camera.

4. Monitor.

5. Cold light source.

6. Middle ear surgery instruments.

7. Power suction machine.

This study was done in the Department of Anatomy, Govt. Medical College, Bettiah during the period of July 2014 - June 2016 on 34 temporal bones. External auditory canal of cadaver was cleaned by fresh water and power suction machine. The dissection was done through transcanal access. Tympanomeatal flap was elevated at posterior meatal wall from 12'o clock to 7'o clock position. Precaution was taken not 
to injure skin of posterior meatal wall and tympanic membrane. Tympanic membrane was carefully dissected from handle of malleus. Now tympanomeatal flap was positioned anteriorly on the anterior meatal wall. Structures of middle ear like chorda tympani nerve, handle of malleus, opening of Eustachian tube, Promontory, Pyramids with tendon of stapedius muscle, facial recess, sinus tympani, ponticulus subiculum and round window were examined. Now epitympanic area was dissected with the help of curettage for proper visualisation of head of malleus, body and short process of incus. Finally, all structures of middle ear cavity were examined under 0 degree and 45 degrees endoscope. Middle ear ossicles was removed and then medial wall was inspected carefully with 0 degree and 45 degrees endoscope. In different cadavers, different types of sinus tympani was observed.

\section{RESULTS}

The hand control of endoscope was difficult, because it should be held by left hand. The coordination to do dissection by observing on monitor needs some practice, because the visualised area is two dimensional so the depth of area should be guessed by repeated practice. The visualisation of middle ear structures was excellent, even those areas which was hidden behind a structure can be visualised by 45 degree endoscope. So, it needs minimum dissection to visualise a particular structure. Out of 34 temporal bones, 2 bones had deep sinus tympani which was hidden posteriorly behind pyramid. Such type of sinus tympani could not be visualised by operating microscope.

\section{Tabular Column}

\begin{tabular}{|c|c|}
\hline Number of temporal bones dissected & 34 \\
\hline Tympanic membrane found intact & 30 \\
\hline $\begin{array}{c}\text { Number of temporal bones in which all the } \\
\text { ossicles found intact }\end{array}$ & 28 \\
\hline $\begin{array}{c}\text { Number of temporal bones in which all } \\
\text { structures of middle ear cavity visualised }\end{array}$ & 31 \\
\hline $\begin{array}{c}\text { Number of temporal bones in which Type A } \\
\text { sinus tympani found }\end{array}$ & 24 \\
\hline $\begin{array}{c}\text { Number of temporal bones in which Type B } \\
\text { sinus tympani found }\end{array}$ & 2 \\
\hline $\begin{array}{c}\text { Number of temporal bones in which Type C } \\
\text { sinus tympani found }\end{array}$ & $\begin{array}{c}\text { Damaged } \\
\text { during } \\
\text { dissection }\end{array}$ \\
\hline Causes of non-visualisation of structures
\end{tabular}

Figures showing the clear view of middle ear structures as well as those hidden areas, which was impossible to visualise by operating microscope due to its linear field of vision.

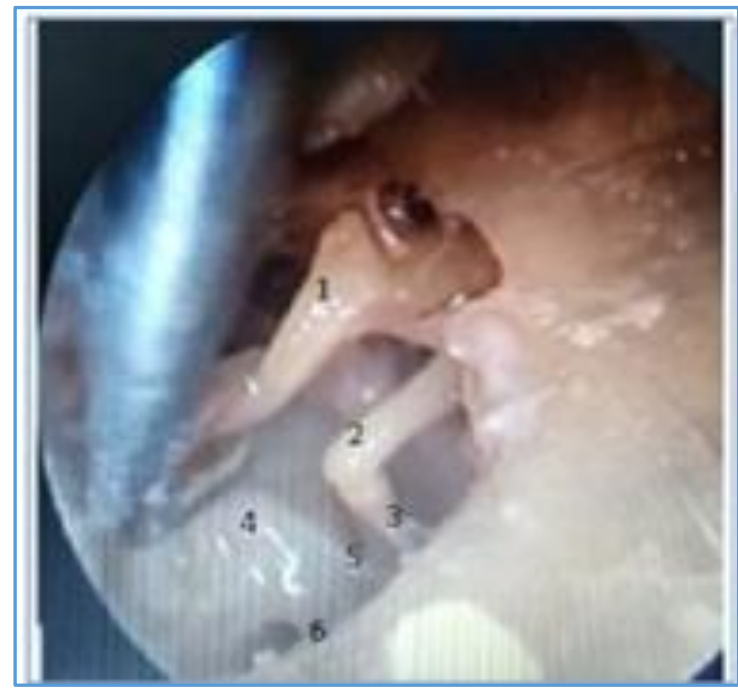

Figure 1. 1. Handle of Malleus, 2. Long Process of INCUS, 3. Stapes, 4. Promontory, 5. Ponticulus, 6. Round Window

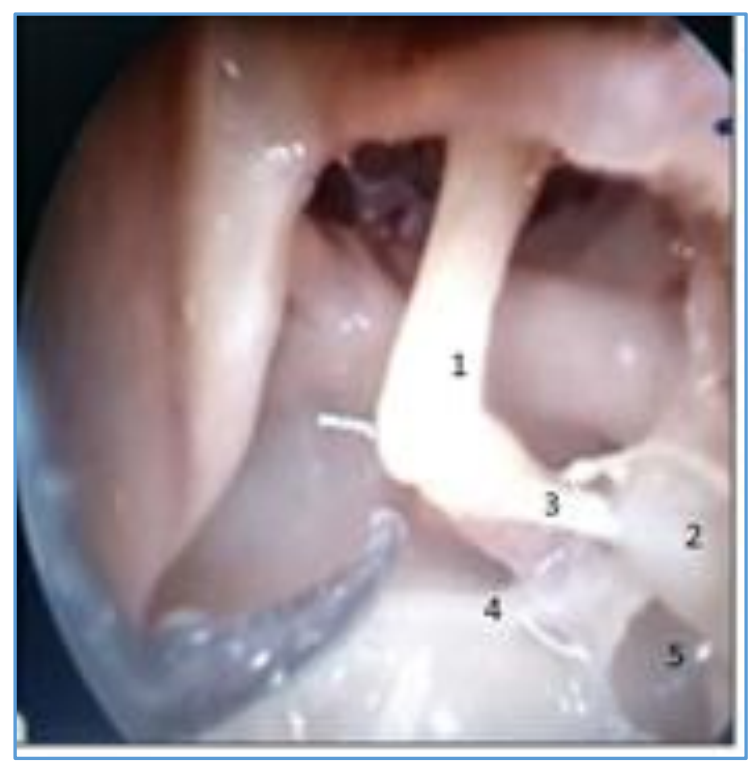

Figure 2. 1. Long process of incus, 2. Pyramid, 3. Stapedius Tendon, 4. Ponticulus, 5. Sinus tympani

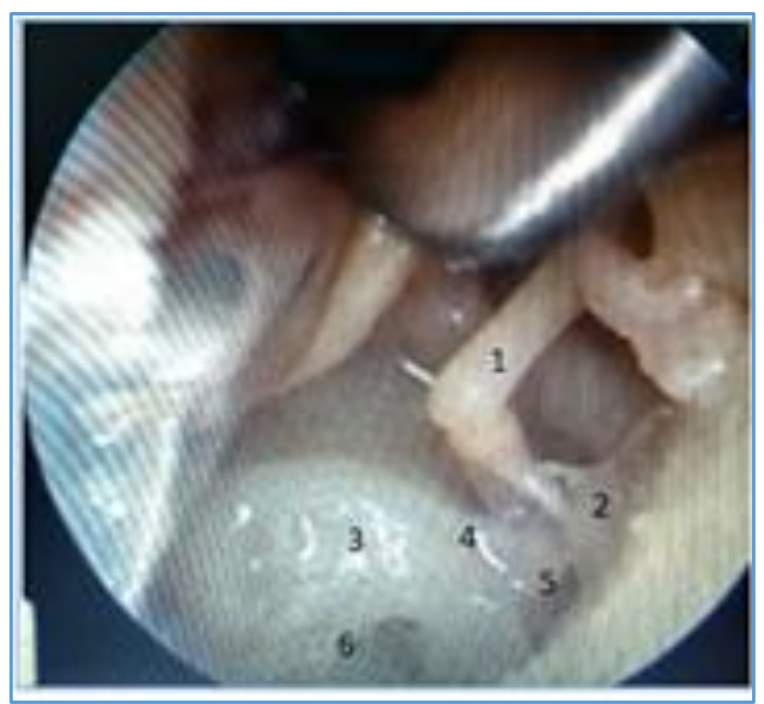

Figure 3. 1. Long process of incus, 2. Pyramid, 3. Promontory, 4. Ponticulus, 5. Sinus Tympani, 6. Round window 


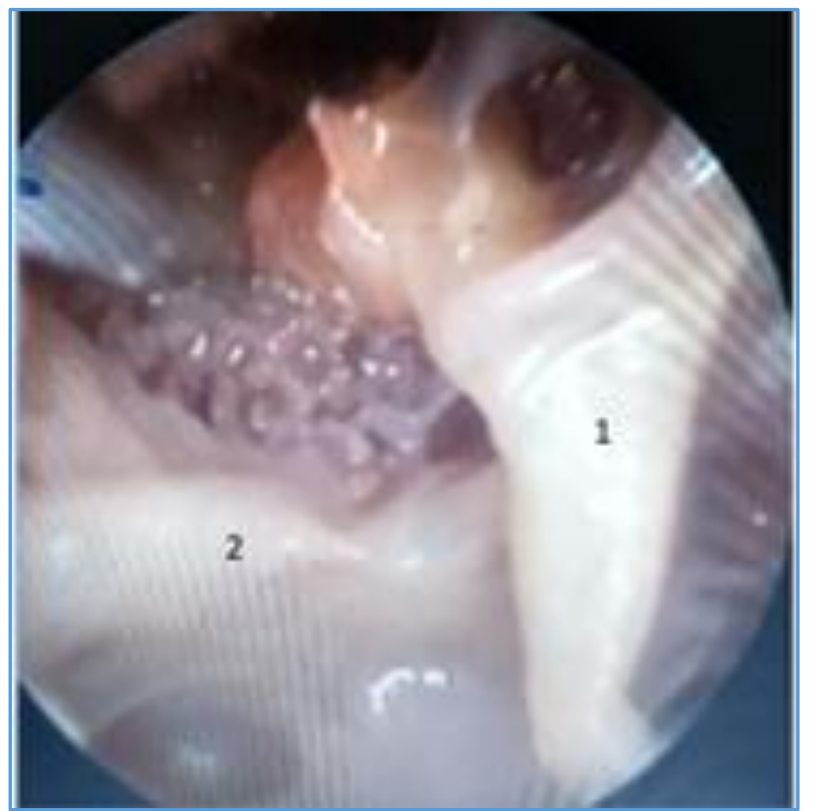

Figure 4. 1. Handle of Malleus, 2. Chorda tympani nerve

\section{DISCUSSION}

Temporal bone dissection on cadaver with help of operating microscope was advocated by many authors for better understanding of anatomy of ear and for improvement of surgical skill. Many otologists committed that they take advantage of microscopic temporal bone dissection on cadaver to improve their surgery. The practice of dissection on cadaver improves hand control on microdrill, surgical instruments and adjusting microscopic vision during surgery. Similarly use of endoscope on cadaver certainly improves the hand control on endoscope and coordination of dissection by looking at the monitor. Probably, first endoscope was invented by Philipp Bozzini two centuries ago. The invention of endoscopic technique in recent time came from urologists who started endoscopic exploration of the urinary bladder with Max Nitze (1849 - 1906). In early stage, it was used for the purpose of diagnostic tools in otology. Use of an endoscope to examine the middle ear cavity was described by Mer in 1967. In early stages, it was used to provide information regarding pathology in the epitympanum, mesotympanum and mastoid antrum and to check the assessment of ossicular chain mobility. ${ }^{5}$ Nowadays, endoscope can be used as diagnostic purposes or as a complementary during microscopic ear surgery for visualisation of difficult areas or solely operating method for tympanoplasty. Some authors prefer use of endoscope for removal of cholesteatoma, because it requires limited dissection. $6,7,8,9$

During the endoscopic dissection, the focus of anatomical areas are those which was poorly visualised by operating microscope. Such areas are retrotympanic spaces. The retrotympanic spaces is a complex structure lying in the posterior aspect of the tympanic cavity. Four spaces present in retrotympanum, two of them lying in medial and anterior and two spaces lying lateral and posterior to the vertical part of facial nerve and pyramid. Pyramid is the fulcrum of the retrotympanic spaces. These spaces are difficult to visualise with operating microscope. The sinus tympani is most important retrotympanic spaces. ${ }^{10}$

The sinus tympani lies medial to the pyramid, stapedius muscle and vertical part of facial nerve. The superior limit of this space is represented by the ponticulus. Presence of disease in sinus tympani is very difficult to remove by operating microscope. The sinus has a great variability in size and shape. Some authors studied the depth of the sinus tympani and described the posterior extension of the sinus tympani. In some cases, there was deep sinus tympani with posterior extension medial to the pyramid and vertical part of facial nerve. Presence of the disease in such kind of sinus tympani does matter, because it is impossible to remove the disease from here under operating microscope. Due to two important risks, complete exploration of medial boundary of sinus tympani is very important. First is the persistence of disease inside the sinus due to incomplete removal and the second is increased risk for ossicular discontinuity and loss of hearing due to cholesteatoma within the sinus tympani. The answer to overcome these difficulties is maximum exposure of the sinus tympani and complete removal of the diseases are essential. With the help of endoscopic surgery, the author suggested the use of endoscopes with different angles to explore the sinus tympani especially 30,45 and 70 degrees endoscopes.

Baki et al studied the endoscopic anatomy of the sinus tympani and found that the sinus tympani is bounded laterally by a constant ledge of bone anterior to the facial nerve. There is deep extension of the sinus tympani posterior to the facial nerve in some cases. In these cases, it was not possible to clearly visualise this region also with endoscope because the orifice plane was perpendicular to the external auditory canal axis.

On the basis of Shape, Sinus Tympani may be

1. Classical Shape- The sinus is located between the ponticulus and subiculum lying medial to the vertical part of facial nerve and to the pyramid.

2. Confluent Shape-An incomplete ponticulus is present and the sinus tympani is confluent to the posterior sinus.

3. Partitioned Shape- A ridge of bone standing from the vertical part of facial nerve to the promontory area is present separating the sinus tympani into two portions: Superior and inferior.

4. Restricted Shape- When a high jugular bulb is present thus reducing the inferior extension of the sinus tympani.

\section{On the Radiological Basis, Sinus Tympani can be Classified into Three Types}

Type A-A small sinus tympani: The medial limit of the vertical portion of the facial nerve corresponded to the depth of the sinus. In these cases, sinus tympani is small and do not present a medial and posterior extension to the facial nerve.

Type B- Deep sinus tympani: The medial boundary of the sinus tympani lies medially with respect to the vertical portion of the facial nerve and do not present a posterior extension to the facial nerve.

Type C- Deep sinus tympani is posterior extension. The medial boundary of the sinus tympani lies medial and posterior to the vertical portion of the facial nerve. In these cases, sinus tympani is very large and deep and all these patients have a well-developed mastoid. 
The other posterior tympanic spaces are: Posterior tympanic sinus which is present superiorly to sinus tympani, Facial recess which is present to vertical part of facial nerve and medial to chorda tympani nerve, Lateral tympanic sinus which is present inferior to facial recess.

The structure present in epitympanic spaces is also well visualised by endoscope with limited dissection. Palva and Coll studied the anatomy of epitympanic compartments and described the anatomy of epitympanic diaphragm studying ventilation pathways of epitympanum. Epitympanic diaphragm includes three malleal ligamental folds (Anterior, Lateral and Posterior), the posterior incudal ligamental fold and two membranous folds (The tensor fold and the lateral incudomalleal fold) together with the malleus and incus. Such type of detailed study is possible with endoscopic dissection with limited disturbing the anatomy of middle ear.

Comparison with other Studies also Prove the above Facts,

1. Joao Flavio Nogueira Junior and Daniel Nogueira Cruz (Resident doctors of Otorhinolaryngologist at the Federal University of Sao Paulo) had dissected 10 temporal bones in May 2009 and their results were:

1. No major technical difficulty performing dissections.

2. They could identify several key structures such as incudomalleolar articulation, tympanic segment of the facial nerve, lateral semicircular canal and aditus ad antrum, all without provoking lesions in the middle ear structures.

2. Daniele Marchioni, Gabriele Molteni and Livio Presutti had studied endoscopic anatomy of the middle ear during 2009 and described clear view of different structures of middle ear cavity (Indian Journal of Otolaryngol and Head and Neck Surgery). They also found that study of anatomy of middle ear cavity with endoscope was useful for better understanding of middle ear anatomy.

\section{CONCLUSION}

Temporal bone dissection on cadaver by endoscope certainly improves the knowledge of anatomy of middle ear cavity so that it improves the surgical skill of otologist. The proper visualisation of those areas of middle ear cavities, which are poorly visualised by operating microscope certainly gives confidence to otologists during endoscopic surgery. Without good knowledge of the anatomy, it is impossible to perform ear surgery and understand ear physiology. Many dissection studies have been done in the past by many authors who described very well the bony structures in the middle ear. Some authors focussed his attention to the tympanic folds and the ventilation pathways. According to some authors, endoscopy of middle ear is the best way to explore the tympanic cavity and to understand the importance of ventilation roots, because disturbance of ventilation roots are the most important factors for initiation of pathology in the middle ear. The goal of surgery in the chronic pathology of the middle ear should be restoration of normal ventilation of the middle ear cavity. So, otologists should do regular endoscopic dissection on cadaver for better understanding of anatomy of middle ear cavity and to learn how to coordinate surgical skill by viewing monitor. Anatomy Department of every Medical College should have facility of endoscopic dissection of temporal bone for the improvement of surgical skills of otologists.

\section{REFERENCES}

[1] Tarabichi M. Endoscopic management of cholesteatoma: long term results. Otolaryngol Head Neck Surg 2000;122(6):874-81.

[2] Tarabichi M. Endoscopic management of acquired cholesteatoma. Am J Otol 1997;18(5):544-9.

[3] Tarabichi M. Endoscopic middle ear surgery. Ann Otol Rhinol Laryngol 1999;108(1):39-46.

[4] Tarabichi M. Endoscopic management of limited attic cholesteatoma. Laryngoscope 2004;114(7):1157-62.

[5] Higgins TS, Courtemanche C, Karakla D, et al. Analysis of transnasal endoscopic versus transseptal microscopic approach for excision of pituitary tumors. Am J Rhinol 2008;22(6):649-52.

[6] McKennan KX. Endoscopic 'second look' mastoidoscopy to rule out residual epitympanic/mastoid cholesteatoma. Laryngoscope 1993;103(7):810-4.

[7] Rosenberg SI, Silverstein H, Willcox TO, et al. Endoscopy in otology and neurotology. Am J Otol 1994;15(2):168-72.

[8] Yung MW. The use of middle ear endoscopy: has residual cholesteatoma been eliminated? J Laryngol Otol 2001;115(12):958-61.

[9] el-Guindy A. Endoscopic transcanal myringoplasty. J Laryngol Otol 1992;106(6):493-5.

[10] Barakate M, Bottrill I. Combined approach tympanoplasty for cholesteatoma: impact of middleear endoscopy. J Laryngol Otol 2008;122(2):120-4. 